\title{
The dynamics of the 1:2 resonant motion with Neptune in the 3D elliptic restricted three-body problem
}

\begin{abstract}
T. A. Kotoulas
University of Thessaloniki, Department of Physics 54124 Thessaloniki, Greece

e-mail: tkoto@skiathos.physics.auth.gr

Received 6 February 2004 / Accepted 30 July 2004

Abstract. We study the planar and the three-dimensional 1:2 resonant motion with Neptune (N1:2) in the framework of the elliptic restricted three-body problem. We examine the dynamics at the 1:2 mean motion resonance with Neptune in a hierarchy of models: the planar circular, the planar elliptic, the three-dimensional circular and the three-dimensional elliptic restricted three body problem. We start from the planar circular model in which we compute families of symmetric periodic orbits with stable and unstable segments. Using the circular planar model as the basic model, we proceed to more realistic models, namely the planar elliptic and the 3D circular problem. Families of symmetric periodic orbits of the above models bifurcate from the families of periodic orbits of the planar circular problem. Moreover, we compute families of symmetric periodic orbits in the 3D elliptic restricted three-body problem. These new families bifurcate from the families of periodic orbits of the planar elliptic and the 3D circular problem. The stability of all orbits is studied and the structure of the phase space is discussed in detail.
\end{abstract}

Key words. celestial mechanics - minor planets, asteroids - Kuiper Belt

\section{Introduction}

The Solar System beyond Neptune consists of a large population of small primordial bodies called Egdeworth-Kuiper belt (E-K) objects or trans-Neptunian objects (TNO's) (see for instance Jewitt 1999; Jewitt \& Luu 2000). Many of them have been discovered at the $2: 3$ resonant region and a few objects have been found close to the 1:2 mean motion resonance with Neptune.

Several studies of the dynamics of the Edgeworth-Kuiper belt have been made up to now: The first theoretical approach to the dynamics of the Kuiper belt was performed by Knežević et al. (1991), who analytically computed the location of the main secular resonances from 2 to $50 \mathrm{AU}\left(a_{\mathrm{N} 1: 2}=47.777 \mathrm{AU}\right)$. Many works based on numerical integrations were done to understand the dynamical structure of the Kuiper belt (Levison \& Duncan 1993; Holman \& Wisdom 1993; Duncan et al. 1995). Especially, in the case of 1:2 resonance with Neptune, a large number of papers has been devoted to the dynamics of this resonance (Beaugé 1994; Beaugé \& Ferraz-Mello 1994; Morbidelli et al. 1995; Malhotra 1996; Gallardo \& Ferraz-Mello 1997; Winter \& Murray 1997; Melita \& Brunini 2000; Nesvorný \& Roig 2001). Reviews of the dynamical structure of the E-K belt and the origin of the Jupiter family of comets were presented by Morbidelli (1999) and Jewitt (1999).

It is known that inside the 1:2 mean motion resonance, as well as in higher order resonances of type $1: \mathrm{k}, k>2$, there are several libration modes (Message 1958; Schubart 1964). Resonant orbits may alternate among the different libration modes, exhibiting a chaotic behavior which may drive diffusion towards large eccentricity. The most regular orbits in the N1:2 resonance are those in a small-amplitude asymmetric libration mode (Morbidelli et al. 1995). It is however possible that, due to the peculiar dynamical structure of the 1:2 resonance, a significant fraction of the captured orbits could be unstable on billion year time-scales, thus reducing the number of surviving objects (Morbidelli 1999). An interesting dynamical characteristic of the 1:2 resonance is the existence of asymmetric librations (Beaugé 1994; Malhotra 1996; Winter \& Murray 1997; Chiang \& Jordan 2002). The existence of asymmetric librations and their importance for the stability of the 1:2 and 1:3 resonant motion in satellite and extrasolar planetary systems has recently shown by Beaugé et al. (2003) and Ferraz-Mello et al. (2003). Lee \& Peale (2003) and Lee (2004) have also done extensive work on this topic. A detailed analysis of asymmetric periodic orbits in 1:2, 1:3 and 1:4 exterior resonances with Neptune was made by Voyatzis et al. (2004).

In the present paper we start from the planar circular model and then we proceed to the planar elliptic (non-zero eccentricity of Neptune) and 3D circular problem in which families of symmetric periodic orbits of the small body are computed. A detailed analysis of families of periodic orbits in the planar circular restricted problem and their stability was performed by Hadjidemetriou \& Ichtiaroglou (1984) for $\mu=$ 0.001 . Moreover, a review of different types of periodic orbits is given in Hadjidemetriou (1988). An atlas of the planar circular restricted three-body problem was produced by 
Winter \& Murray (1994a,b). Hénon (1997) made a systematic study of generating families, which are the limits of families of periodic orbits in the restricted three-body problem when the mass ratio of the two main bodies becomes vanishingly small.

In the planar elliptic problem, the families of periodic orbits bifurcate from the families of periodic orbits of the planar circular problem at those points where the period is a multiple of the period of Neptune (in the inertial frame). A systematic study of the resonant structure of the restricted three-body problem for the Sun-Jupiter-asteroid system in the plane was made by Hadjidemetriou (1993). Periodic orbits in the external resonances in the planar elliptic restricted three-body problem were computed by Varadi (1999) and Haghighipour et al. (2003).

Broucke (2001) made an extensive numerical study of orbits of planets of a binary star system in the three-dimensional restricted problem. A detailed analysis of symmetric periodic orbits of the 3D circular problem (CR3BP) at the exterior resonances 1:2, 2:3 and 3:4 was made by Kotoulas \& Hadjidemetriou 2002 (hereafter called KH2002). Basing our work on the latter paper, we proceed to the computation of the families of periodic orbits of the 3D elliptic restricted threebody problem (ER3BP). The new three-dimensional families of symmetric periodic orbits in the elliptic problem bifurcate from the families of the 3D circular problem at those points where the period is a multiple of the period of Neptune (in the inertial frame) or from vertical critical orbits of the corresponding planar elliptic problem (Sect. 4). Their stability is also studied.

The Poincaré mapping of the ER3BP is six-dimensional. The existence of fixed points is an important feature of the mapping. These correspond to periodic orbits of the system. Moreover, their position and stability determine critically the topology of the phase space. This clarifies why it is important to know the families of symmetric periodic orbits. Thus, the dynamics inside the 1:2 resonance with Neptune is still an open subject of debate and we believe that our work contributes to the understanding of this problem.

\section{The Lagrangian for the motion of a small body}

We shall use a rotating frame of reference Oxyz whose $x$-axis is the line joining the Sun, S, with Neptune, N, the positive direction being from $S$ to $N$, its origin is at their centre of mass, the $y$-axis is in the orbital plane of Neptune and the $z$-axis is perpendicular to the $x y$ plane. In the usual normalized units the semimajor axis of Neptune's heliocentric orbit $=1$, the gravitational constant is $G=1$ and the total mass $m_{\mathrm{S}}+m_{\mathrm{N}}=1$. For the theory of the restricted three-body problem see Szebehely (1967) or Roy (1982). In the general case, where the orbit of Neptune is elliptic, the angular velocity of rotation of the Oxyz frame is not constant and the positions of the Sun and Neptune on the rotating $x$-axis are not fixed.

The Lagrangian of a small body in the rotating frame, in the units mentioned above, is:

$$
L=0.5\left[(\dot{x}-\dot{\theta} y)^{2}+(\dot{y}+\dot{\theta} x)^{2}+(\dot{z})^{2}\right]+\frac{1-\mu}{r_{1}}+\frac{\mu}{r_{2}},
$$

where

$r_{1}=\sqrt{(x+\mu r)^{2}+y^{2}+z^{2}}$

$r_{2}=\sqrt{[x-(1-\mu) r]^{2}+y^{2}+z^{2}}$.

The distance, $r$, between Sun and Neptune and the polar angle, $\theta$, which will be taken as the true anomaly of Neptune, are known functions of time. For computational purposes we have computed the differential equations in the variables $x, y, z$ obtained from Eq. (1), together with the differential equations in $r, \theta$ of the two-body problem obtained from the Lagrangian:

$L=0.5\left(\dot{r}^{2}+r^{2} \dot{\theta}^{2}\right)+\frac{1}{r}$.

In the particular case of circular motion of Neptune, we have $r=1$ and $\dot{\theta}=n^{\prime}=1$ in normalized units.

In the above mentioned rotating frame Oxyz there exist families of symmetric periodic orbits for the small body which "lie" outside the orbit of Neptune. In this work we shall consider direct periodic orbits for the small body, i.e. the TNO revolves around the Sun in the same direction as Neptune (with respect to the inertial frame). For the mass of Neptune we have chosen the value $\mu=5.178 \times 10^{-5}$ and the orbital period of Neptune around the Sun, in the normalized units we use, is equal to $2 \pi$.

\section{The planar restricted three-body problem}

The study of the planar restricted three-body problem at the 1:2 mean motion resonance with Neptune will be done with the computation of periodic orbits. Other known methods are: averaged Hamiltonians and mapping models. The periodic orbits are of particular interest since they define the structure of the associated resonance. In this section we shall present families of periodic orbits in the planar restricted three-body problem and study their stability type.

\subsection{The circular case}

In this paragraph we shall deal with symmetric periodic orbits. This means that the initial conditions of a periodic orbit are:

$x(0)=x_{0}, y(0)=0, \dot{x}(0)=0, \dot{y}(0)=\dot{y}_{0}$.

At half the period $T$ of the orbit we must have:

$y\left(x_{0}, \dot{y}_{0} ; T / 2\right)=0, \dot{x}\left(x_{0}, \dot{y}_{0} ; T / 2\right)=0$.

Equations (5) give the periodicity conditions for a symmetric periodic orbit in the planar circular problem. A symmetric periodic orbit of multiplicity $p$ starts perpendicularly from the $x$-axis and again crosses the $x$-axis perpendicularly after $p$ intersections in the same direction. There are two families of periodic orbits for the 1:2 resonance, symmetric with respect to the $x$-axis, one of them corresponding to the small body at perihelion at $t=0$ (Family I) and the other to the small body at aphelion (Family II). Each member of the family is defined by its initial conditions $\left(x_{0}, \dot{y}_{0}\right)$, or equivalently, by $\left(x_{0}, h\right)$, where $h$ is the Jacobi-constant. Thus, a monoparametric family 

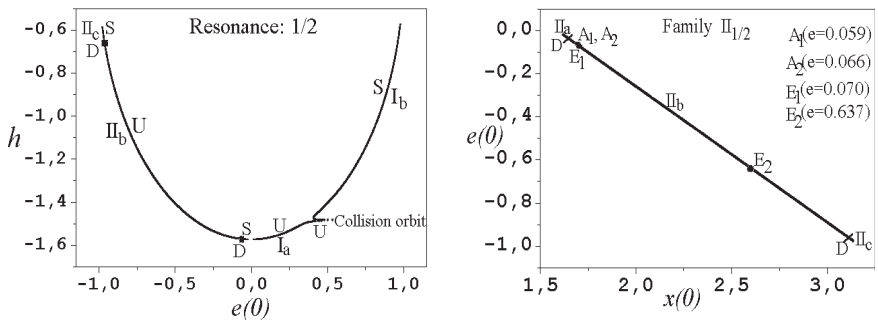

Fig. 1. a) The families I and II of 1:2 resonant periodic orbits $(\mu>0)$ in the plane $e(0)-h$ where $h$ is the Jacobi-constant. The gap at the resonance is due to the non-continuation of the circular orbits. At point $\mathrm{D}$ the stability type changes. The small body is at perihelion $(e(0)>0)$ or at aphelion $(e(0)<0)$ at $t=0$. b) Family II of symmetric periodic orbits at the plane $x(0)-e(0)$. The bifurcation points from the planar circular problem to the planar elliptic $\left(E_{1}, E_{2}\right)$ and to 3D circular one $\left(\mathrm{A}_{1}, \mathrm{~A}_{2}\right)$ are also indicated. The symbols " $\mathrm{S}$ " and " $\mathrm{U}$ " denote stability and instability respectively.

of symmetric periodic orbits is defined by a smooth curve in the plane $x_{0}-h$ of initial conditions, which is called the "characteristic curve". Along each family the eccentricity of the small body increases starting from zero. These families are shown in Fig. 1a (for other diagrams see KH2002).

Periodic orbits of the family $\mathrm{I}_{1: 2}$ start with multiplicity $p=1$. In the family $\mathrm{I}_{1: 2}$ we have a collision orbit with Neptune when the eccentricity satisfies the relation $a(1-e)=1$, where $a$ is the semimajor axis of the corresponding resonant orbit. This collision orbit occurs at $e=0.37$. The family $\mathrm{I}_{1: 2}$ starts out unstable; after the collision orbit the multiplicity of periodic orbits changes from 1 to 2 and this family becomes stable. So, the family $\mathrm{I}_{1: 2}$ consists of two parts: $\mathrm{I}_{\mathrm{a}}$ (unstable), $\mathrm{I}_{\mathrm{b}}$ (stable) (Fig. 1a).

In the family $\mathrm{II}_{1: 2}$, we start with multiplicity $p=1$ but the multiplicity changes from 1 to 2 at $e=0.312$. No collision orbits appear in this family. Family $\mathrm{II}_{1: 2}$ starts with stable orbits until a certain value of the eccentricity $\left(e_{1}=0.035\right)$ and then the orbits become unstable. At a very high value of the eccentricity $\left(e_{2}=0.96\right)$ the family $\mathrm{II}_{1: 2}$ again becomes stable. So, family $\mathrm{II}_{1: 2}$ consists of three parts: $\mathrm{II}_{\mathrm{a}}$ (stable), $\mathrm{II}_{\mathrm{b}}$ (unstable) and $\mathrm{II}_{\mathrm{c}}$ (stable) (Fig. 1b). The stability of periodic orbits has been studied many times in the past (Hénon 1965; Hadjidemetriou 1988; Celletti et al. 2002). The transition from stability to instability and vice versa in the family $\mathrm{II}_{1: 2}$ coincides with the bifurcation to asymmetric librations. These were studied by Beaugé (1994), Morbidelli et al. (1995), Malhotra (1996), Winter \& Murray (1997) and Chiang \& Jordan (2002). Moreover, asymmetric librations are associated with asymmetric periodic orbits (Voyatzis et al. 2004).

\subsection{The elliptic case}

We briefly state some basic facts. For the details of computing periodic orbits in this problem and their stability, see Broucke $(1968,1969)$. Families of periodic orbits of the planar ER3BP were computed by Kribbel \& Dvorak (1988) and Hadjidemetriou (1992, 1993).

We have two degrees of freedom, as in the circular problem, but now the system is non-autonomous. The distance between the Sun and Neptune is not constant now and the $x \mathrm{O} y$ frame is not a uniformly rotating system. The initial conditions also involve the position and velocity of the Sun and Neptune at $t=0$. For a symmetric periodic orbit of the small body we get:

$y(0)=0, \dot{x}(0)=0, \dot{r}(0)=0, \theta(0)=0, \pi$

with an indication that Neptune is at perihelion or aphelion at $t=0$. The nonzero initial conditions are then: $x(0)=x_{0}$, $\dot{y}(0)=\dot{y}_{0}$ and also $r(0), \dot{\theta}(0)$, which, however, are determined from Keplerian theory in terms of the eccentricity of Neptune, taking into account that the semimajor-axis of the Sun-Neptune system is equal to one. Thus, the eccentricity of Neptune $\left(e_{\mathrm{N}}\right)$ appears as a parameter of the problem. By varying $e_{\mathrm{N}}$ we have a family of periodic orbits of the elliptic problem. For $\dot{r}(0)=0$ and $\theta(0)=0$ or $\pi$ the periodicity conditions are now:

$y\left(x_{0}, \dot{y}_{0} ; e_{\mathrm{N}} ; T / 2\right)=0, \dot{x}\left(x_{0}, \dot{y}_{0} ; e_{\mathrm{N}} ; T / 2\right)=0$

where $T$ is the period of the periodic orbit in the rotating frame. Due to the fact that at $t=T / 2$ we must have $\dot{r}=0$ and $\theta=0, \pi$ too, it arises that the period $T$ must necessarily be equal to $2 \pi$ (or a multiple of it). In the normalized units $\left(m_{\mathrm{S}}+m_{\mathrm{N}}=1, G=\right.$ $1, a_{\mathrm{N}}=1$ ) the period of Neptune is equal to $2 \pi$. This means that the periodic orbits in the elliptic problem in the rotating frame are also periodic in the inertial frame, in contrast to the circular case where, in general, this is not true.

The bifurcation for elliptic periodic orbits is at the point where the period is exactly equal to $4 \pi$. There are two bifurcation points in the family $\mathrm{II}_{1: 2}: E_{1}(e=0.07)$ and $E_{2}(e=0.637)$ (Fig. 1b). So, these points give rise to two pairs of families of periodic orbits in the planar elliptic problem. The first pair is: $\mathrm{E}_{1 \mathrm{p}}^{1: 2}, \mathrm{E}_{1 \mathrm{a}}^{1: 2}$ and the second: $\mathrm{E}_{2 \mathrm{p}}^{1: 2}, \mathrm{E}_{2 \mathrm{a}}^{1: 2}$. In the families $\mathrm{E}_{1 \mathrm{p}}^{1: 2}, \mathrm{E}_{2 \mathrm{p}}^{1: 2}$ Neptune is at perihelion at $t=0$ and in the families $\mathrm{E}_{1 \mathrm{a}}^{1: 2}, \mathrm{E}_{2 \mathrm{a}}^{1: 2}$ Neptune is at aphelion at $t=0$. The numerical computations have revealed that the families $\mathrm{E}_{1 \mathrm{a}}^{1: 2}$ and $\mathrm{E}_{2 \mathrm{a}}^{1: 2}$ merge at $e_{\mathrm{N}}=0.24$. All are unstable (KH2002). These families are also presented in Fig. 2.

Families of periodic orbits in the 3D ER3BP bifurcate from the vertical critical orbits of the planar elliptic problem. So, we computed the vertical stability of the families $\mathrm{E}_{1 \mathrm{p}}^{1: 2}, \mathrm{E}_{2 \mathrm{p}}^{1: 2}, \mathrm{E}_{1 \mathrm{a}}^{1: 2}$, $\mathrm{E}_{2 \mathrm{a}}^{1: 2}$. We found that there is one vertical critical orbit in the family $\mathrm{E}_{1 \mathrm{p}}^{1: 2}$. It is $\mathrm{M}_{1}\left(e=0.218, e_{\mathrm{N}}=0.474\right)$ (Fig. 2a). This vertical critical orbit can give rise to a family of periodic orbits at the 3D ER3BP (Sect. 4.2). On the other hand, families $E_{1 \mathrm{a}}^{1: 2}$, $\mathrm{E}_{2 \mathrm{p}}^{1: 2}$ and $\mathrm{E}_{2 \mathrm{a}}^{1: 2}$ are vertically stable (Figs. $2 \mathrm{~b}-\mathrm{c}$ ). In Figs. $2 \mathrm{a}-\mathrm{c}$ we have used the parameter $b_{2}=\left(2 \alpha_{v}-2\right) \times 10^{6}$ for the vertical axis, instead of the vertical stability index $\alpha_{v}$ (Hénon 1973) of the periodic orbits of the elliptic problem. This help us to have a clear view of the results when $\alpha_{v}$ is close to 1 . We note here that a periodic orbit is vertically critical when $\alpha_{v}=1$ or $b_{2}=0$.

The majority of periodic orbits in the planar elliptic problem is unstable for the of 1:2 resonance (Haghighipour et al. 2003). Haghighipour et al. (2003) made an extensive numerical study of the periodic orbits of planar, elliptic restricted three-body planetary systems consisting of a star, an inner massive planet and an outer massless body in the external 1:2 mean motion resonance. But for certain values of the mass and the 

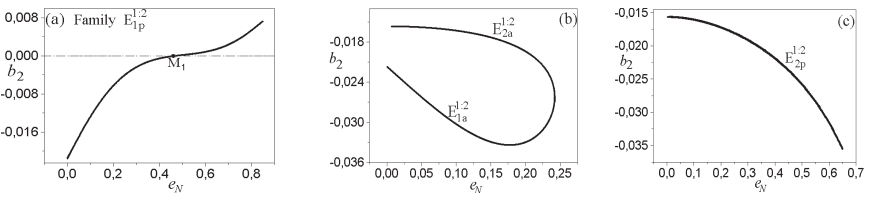

Fig. 2. Diagrams of the parameter $b_{2}$-eccentricity of the small body for all the families of periodic orbits of the planar elliptic problem: a) family $\mathrm{E}_{1 \mathrm{p}}^{1: 2}$; b) $\mathrm{E}_{1 \mathrm{a}}^{1: 2}$ and $\mathrm{E}_{2 \mathrm{a}}^{1: 2}$; c) $\mathrm{E}_{2 \mathrm{p}}^{1: 2}$.

orbital eccentricity of the inner planet they found stable periodic orbits. Their results were applied to the 1:2 resonance of the extrasolar planetary system GJ 876 .

\section{The 3D restricted three-body problem}

The 3D elliptic restricted three-body problem is a nonautonomous Hamiltonian system with three degrees of freedom. This model is the most realistic one in the framework of the restricted three-body problem since it describes the motion of the small body in space and the perturber planet is in an elliptic orbit. The study of such systems presents several difficulties (Simó 1995). Skokos (2001) made a complete analysis of the stability of periodic orbits of high dimensional autonomous Hamiltonian systems. Here, as in the planar case, we shall start with the 3D circular case.

\subsection{The 3D circular problem at 1:2 resonance}

The three-dimensional families of symmetric periodic orbits in the circular problem bifurcate from the vertical critical orbits of the corresponding planar circular problem (Hénon 1973; Markellos et al. 1981). Three-dimensional periodic orbits were also studied in the past (e.g. Zagouras \& Markellos 1977; Michalodimitrakis 1979; Broucke 2001). Ichtiaroglou et al. (1978) gave a proof that a vertical critical orbit of the planar restricted three-body problem (circular or elliptic) can be continued to a periodic orbit of the corresponding three-dimensional problem. Moreover, Ichtiaroglou \& Michalodimitrakis (1980) showed the existence of families of 3D periodic orbits which bifurcate from vertical critical orbits of the planar, general or restricted, problem of three bodies.

Checking the vertical stability of the families $\mathrm{I}_{1: 2}$ and $\mathrm{II}_{1: 2}$, we found that the family $\mathrm{I}_{1: 2}$ is vertically stable; vertical critical orbits do exist in the family $\mathrm{II}_{1: 2}$. There are two vertical critical orbits in the family $\mathrm{II}_{1: 2}: \mathrm{A}_{1}(e=0.059)$ and $\mathrm{A}_{2}(e=0.066)$ (Fig. 1b) which can cause bifurcation for the 3D families $A_{1}$ and $\mathrm{A}_{2}$ respectively. A detailed analysis of families of 3D symmetric periodic orbits and their stability was made in KH2002. Here we present briefly the families of these orbits. These results are necessary for the connection between the two models: the 3D circular and the 3D elliptic restricted three-body problem. All the results are referred to the rotating frame of reference Oxyz.

Family $\mathrm{A}_{1}$ is symmetric with respect to the $x z$-plane (type 1) and bifurcates from the first vertical critical orbit ( $e=0.059$ ); family $\mathrm{A}_{2}$ is symmetric with respect to the $x$ axis (type 2$)$ and bifurcates from the second one $(e=0.066)$.
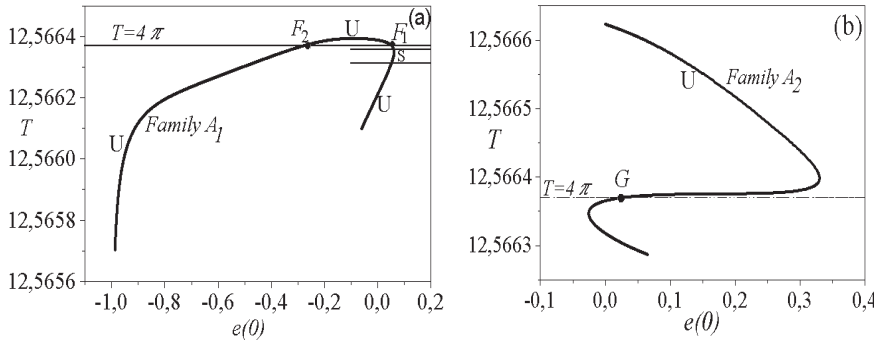

Fig. 3. Resonance 1:2: a) Projection of the family $\mathrm{A}_{1}$ on the $T-$ $e(0)$ plane. b) Projection of the family $\mathrm{A}_{2}$ on the $T-e(0)$ plane. The points $F_{1}, F_{2}$ and $G$ are the bifurcation points from the $3 \mathrm{D}$ Circular to 3D Elliptic Problem $(T=4 \pi)$. The negative values of the eccentricity stand for the case where the small body lies initially at the aphelion of its orbit. The symbols "S" and "U" stand for stability and instability of periodic orbits.

Types 1 and 2 are not defined any longer; they are just types A and $\mathrm{B}$ defined later in Sect. 4.3 with $e_{\mathrm{N}}=0$.

In Fig. 3 we show the projections of the families $A_{1}$ and $A_{2}$ on the planes $T-e(0)$ (period eccentricity) and we indicate the bifurcation points in the $3 \mathrm{D}$ elliptic problem. Both families are unstable; a stable segment exists for the family $\mathrm{A}_{1}$ $\left(55.7^{\circ}<i<63.8^{\circ}\right)$ (Fig. 3a). For the computation of the stability of periodic orbits in systems with 3 degrees of freedom we followed the methods of Broucke (1969), Hadjidemetriou (1975) and Michalodimitrakis (1979).

As we mentioned above, a stable segment exists for the family $\mathrm{A}_{1}\left(55.7^{\circ}<i<63.8^{\circ}\right)$. The critical symmetric periodic orbits in which the instability turns to stability or viceversa may give bifurcation to $3 \mathrm{D}$ unstable asymmetric periodic orbits (an analogous case to that of family $\mathrm{II}_{1: 2}$ of symmetric periodic orbits in the planar circular model). The computation of asymmetric periodic orbits is not so easy. The Poincaré sections are four-dimensional, so we cannot have a clear picture of them as in the case of the planar circular model and we cannot find easily the initial conditions of the asymmetric periodic orbits. Furthermore, asymmetric periodic orbits start from unstable symmetric periodic orbits and numerical difficulties may appear while they are being computed.

On the other hand there are many cases of asteroidal resonances in which bifurcation points from planar circular to planar elliptic model do not exist (Tsiganis et al. 2002a,b). This phenomenon is associated to the specific phase space structure of these resonances. Tsiganis et al. (2002a,b) made a systematic study of medium/high-order mean motion resonances with Jupiter and showed that the occurrence of stable chaos is related to the fact that there do not exist families of periodic orbits in the planar elliptic problem and in the 3D circular problem corresponding to the examined resonance.

\subsection{Methods for finding periodic orbits in the 3D elliptic restricted three-body problem}

(a) Families of periodic orbits of the 3D ER3BP, with the eccentricity of Neptune $\left(e_{\mathrm{N}}\right)$ as a parameter, bifurcate from the families of the 3D CR3BP mentioned above at those orbits whose period is a multiple of $2 \pi$ (in general $T=\frac{p}{q} \pi$ 
Table 1. Bf points from 3D CR3BP to 3D ER3BP.

\begin{tabular}{cccc}
\hline \hline Resonance & Period & Family & Bifurcation Points \\
\hline $1: 2$ & $4 \pi$ & $\mathrm{A}_{1}$ & $\mathrm{~F}_{1}\left(e=0.05, i=80.02^{\circ}\right)$ \\
$1: 2$ & $4 \pi$ & $\mathrm{A}_{1}$ & $\mathrm{~F}_{2}\left(e=0.27, i=113.80^{\circ}\right)$ \\
$1: 2$ & $4 \pi$ & $\mathrm{A}_{2}$ & $G\left(e=0.04, i=101.66^{\circ}\right)$ \\
\hline
\end{tabular}

Table 2. Bf points from 2D ER3BP to 3D ER3BP.

\begin{tabular}{cccc}
\hline \hline Resonance & $b_{2}$ & Family & Bifurcation Points \\
\hline $1: 2$ & 0.0 & $\mathrm{E}_{1 \mathrm{p}}$ & $M_{1}\left(e=0.218, e_{\mathrm{N}}=0.474\right)$ \\
\hline
\end{tabular}

where $p, q$ are integers). Checking the value of the pe$\operatorname{riod} T$ in each family of the 3D circular restricted threebody problem, we found that there exist three periodic orbits in total in the families $A_{1}, A_{2}$ at the resonance 1:2, whose period is equal to $4 \pi$ (Fig. 3 ). The results are summarized in Table 1 (the term $B f$ points stand for bifurcation points, see also KH2002).

(b) The second way to search for periodic orbits that bifurcate to the 3D ER3BP is to check the vertical stability of periodic orbits of the corresponding planar elliptic problem. This was already presented in Sect. 3.2 and the relevant results for the 3D case are shown in Table 2.

\subsection{Families of periodic orbits of the $3 D$ elliptic restricted problem (New Families of symmetric periodic orbits)}

We shall present in the following the families of 3D resonant periodic orbits of the elliptic restricted 3-body problem at the $1: 2$ exterior resonance for $\mu=5.178 \times 10^{-5}$. All the results are referred to the rotating frame of reference Oxyz. The eccentricity and the inclination vary within a family, but the semimajor axis is almost constant, $a_{1: 2} \approx 47.777 \mathrm{AU}$. The angles $\sigma, v, \sigma_{z}$ are defined as:

$\sigma=-\lambda^{\prime}+2 \lambda-\varpi, \sigma_{z}=-\lambda^{\prime}+2 \lambda-\Omega, v=+\lambda^{\prime}-2 \lambda+\varpi^{\prime}(8)$

where $\lambda$ is the mean longitude, $\varpi$ is the longitude of perihelion, $\Omega$ is the longitude of the ascending node of the small body and the primed quantities refer to Neptune.

There are two types of symmetric periodic orbits in the elliptic restricted three-body problem which are studied in the rotating $\mathrm{O} x y z$ frame:

Type A: Symmetric periodic orbits with respect to the xz-plane.

A 3D simple symmetric periodic orbit starts at $t=0$ perpendicularly from the $x z$-plane $(y(0)=0, \dot{x}(0)=0, \dot{z}(0)=$ 0 ) with Neptune at perihelion or aphelion $(\dot{r}(0)=0, \theta=0$ or $\pi)$ and again crosses the $x z$-plane perpendicularly at the first intersection $\left(y\left(x_{0}, \dot{y}_{0}, z_{0} ; e_{\mathrm{N}} ; T / 2\right)=0, \dot{x}\left(x_{0}, \dot{y}_{0}, z_{0} ; e_{\mathrm{N}} ; T / 2\right)=\right.$ $\left.0, \dot{z}\left(x_{0}, \dot{y}_{0}, z_{0} ; e_{\mathrm{N}} ; T / 2\right)=0\right)$ and $(\dot{r}(0)=0, \theta=0$ or $\pi)$, where $T$ is the period of the orbit. Since for a symmetric periodic orbit we have $y(0)=0, \dot{x}(0)=0, \dot{z}(0)=0$ and $(\dot{r}(0)=0$, $\theta=0$ or $\pi)$, the non-zero initial conditions are: $x(0), \dot{y}(0), z(0)$ and $r(0), \dot{\theta}(0)$. Consequently, a symmetric periodic orbit can be represented by a point in the four-dimensional space $x_{0}-\dot{y}_{0}-z_{0}-e_{\mathrm{N}}$. Also, a monoparametric family is generated by varying the eccentricity of Neptune $\left(e_{\mathrm{N}}\right)$.

\section{Type B: Symmetric periodic orbits with respect to the $x$-axis}

A 3D simple symmetric periodic orbit starts at $t=0$ perpendicularly from the $x$-axis $(y(0)=0, z(0)=0, \dot{x}(0)=0)$ with Neptune at perihelion or aphelion $(\dot{r}(0)=0, \theta=0$ or $\pi)$ and again crosses the $x$-axis perpendicularly at the first intersection $\left(y\left(x_{0}, \dot{y}_{0}, \dot{z}_{0} ; e_{\mathrm{N}} ; T / 2\right)=0, z\left(x_{0}, \dot{y}_{0}, \dot{z}_{0} ; e_{\mathrm{N}} ; T / 2\right)=\right.$ $\left.0, \dot{x}\left(x_{0}, \dot{y}_{0}, \dot{z}_{0} ; e_{\mathrm{N}} ; T / 2\right)=0\right)$ and $(\dot{r}(0)=0, \theta=0$ or $\pi)$, where $T$ is the period of the orbit. Since for a symmetric periodic orbit we have $y(0)=0, z(0)=0, \dot{x}(0)=0$ and $(\dot{r}(0)=0, \theta=0$ or $\pi)$, the non-zero initial conditions are: $x(0), \dot{y}(0), \dot{z}(0)$ and $r(0), \dot{\theta}(0)$. Consequently, a symmetric periodic orbit can be represented by a point in the four-dimensional space $x_{0}-\dot{y}_{0}-\dot{z}_{0}-e_{\mathrm{N}}$. Also, a monoparametric family is generated by varying the eccentricity of Neptune $\left(e_{\mathrm{N}}\right)$. Note that the energy integral does not exist in this case.

There are two bifurcation points from the family $\mathrm{A}_{1}$ of the $3 \mathrm{D}$ circular problem to the $3 \mathrm{D}$ elliptic one $(T=4 \pi)$. These are: $\mathrm{F}_{1}\left[e=0.05, i=80.02^{\circ}\right]$ and $\mathrm{F}_{2}\left[e=0.27, i=113.80^{\circ}\right]$. So, two pairs of families of periodic orbits of the $3 \mathrm{D}$ elliptic problem arise which bifurcate from the above $3 \mathrm{D}$ orbits on the family $A_{1}$. One pair is $F_{1 p}^{1: 2}, F_{1 a}^{1: 2}$ and bifurcates from the point $\mathrm{F}_{1}$; the other pair is $\mathrm{F}_{2 \mathrm{p}}^{1: 2}, \mathrm{~F}_{2 \mathrm{a}}^{1: 2}$ and bifurcates from the point $F_{2}$. These families of periodic orbits are of type A. For the families $F_{1 p}^{1: 2}, F_{2 p}^{1: 2}$ Neptune is at perihelion and for the families $\mathrm{F}_{1 \mathrm{a}}^{1: 2}, \mathrm{~F}_{2 \mathrm{a}}^{1: 2}$ it is at aphelion at $t=0$. For the family $\mathrm{F}_{1 \mathrm{p}}^{1: 2}$ we have: $\sigma=0, \sigma_{z}=3 \pi / 2, v=0$ and $\omega=3 \pi / 2, \Omega=\pi / 2$ while for the family $\mathrm{F}_{1 \mathrm{a}}^{1: 2}$ we have: $\sigma=0, \sigma_{z}=3 \pi / 2, v=\pi$ and $\omega=3 \pi / 2, \Omega=\pi / 2$. This is so because the small body is at perihelion at $t=0$. The values of $\sigma, \sigma_{z}, v$ for the families $\mathrm{F}_{2 \mathrm{p}}^{1: 2}$, $\mathrm{F}_{2 \mathrm{a}}^{1: 2}$ are: $\sigma=\pi, \sigma_{z}=3 \pi / 2, v=0$ and $\sigma=\pi, \sigma_{z}=3 \pi / 2, v=\pi$ respectively. This is so because the small body is at aphelion at $t=0$. We also have $\omega=\Omega=\pi / 2$.

\section{(i) The families $F_{1 p}^{1: 2}, F_{2 p}^{1: 2}$}

In Fig. 4 we show the families $F_{1 p}^{1: 2}, F_{1 a}^{1: 2}, F_{2 p}^{1: 2}, F_{2 a}^{1: 2}$ of $3 \mathrm{D}$ periodic orbits in the $x_{0}-z_{0}-e_{\mathrm{N}}$ space. The curve in the $x_{0}-z_{0}$ plane is the projection of the family $\mathrm{A}_{1}$ of the 3D CR3BP. In Figs. $5 \mathrm{a}$ and $5 \mathrm{~b}$ we show the projections of the family $\mathrm{F}_{1 \mathrm{p}}^{1: 2}$ to the $z_{0}-e_{\mathrm{N}}$ and $i(0)-e(0)$ planes. The same is done for the family $F_{2 p}^{1: 2}$ in Figs. $5 c$ and $5 d$. The family $F_{1 p}^{1: 2}$ starts from the orbit $\mathrm{F}_{1}$ and terminates at the collision orbit $\mathrm{K}_{1}[e=0.73$, $\left.i=62.57^{\circ}\right]$ with $e_{\mathrm{N}}=0.913$. Numerical difficulties appear for $e_{\mathrm{N}}>0.913$, where $e_{\mathrm{N}}$ is the eccentricity of Neptune. In this family the small body is initially at perihelion and eccentricity $(e)$ increases continuously starting from $e=0.05$ and reaches the maximum value $e_{\max }=0.73$ at the collision orbit $\mathrm{K}_{1}$. The inclination starts from $i=80.02^{\circ}$, attains a minimum value at $i_{\min }=45.24^{\circ}$ and then increases until $i=62.57^{\circ}$ (Fig. 5b). The stability of the family $\mathrm{F}_{1 \mathrm{p}}^{1: 2}$ is shown in Fig. $5 \mathrm{~b}$. 


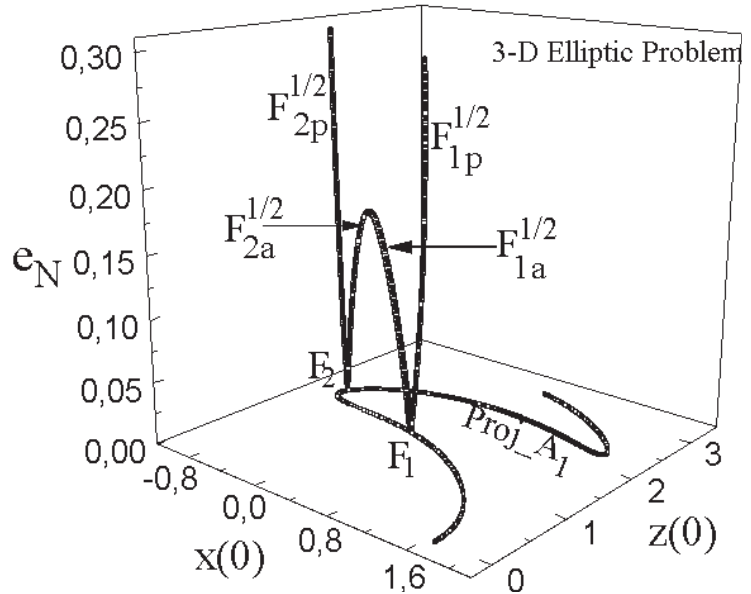

Fig. 4. Resonance 1:2: Projections of the families $F_{1 p}^{1: 2}, F_{2 p}^{1: 2}, F_{1 a}^{1: 2}, F_{2 a}^{1: 2}$ in $x_{0}-z_{0}-e_{\mathrm{N}}$ space, where $e_{\mathrm{N}}$ is the eccentricity of Neptune. The points $F_{1}$ and $F_{2}$ are the starting points of the above families in the 3D ER3BP. The curve in the $x_{0}-z_{0}$ plane is the projection of the family $\mathrm{A}_{1}$ of the $3 \mathrm{D}$ CR3BP.
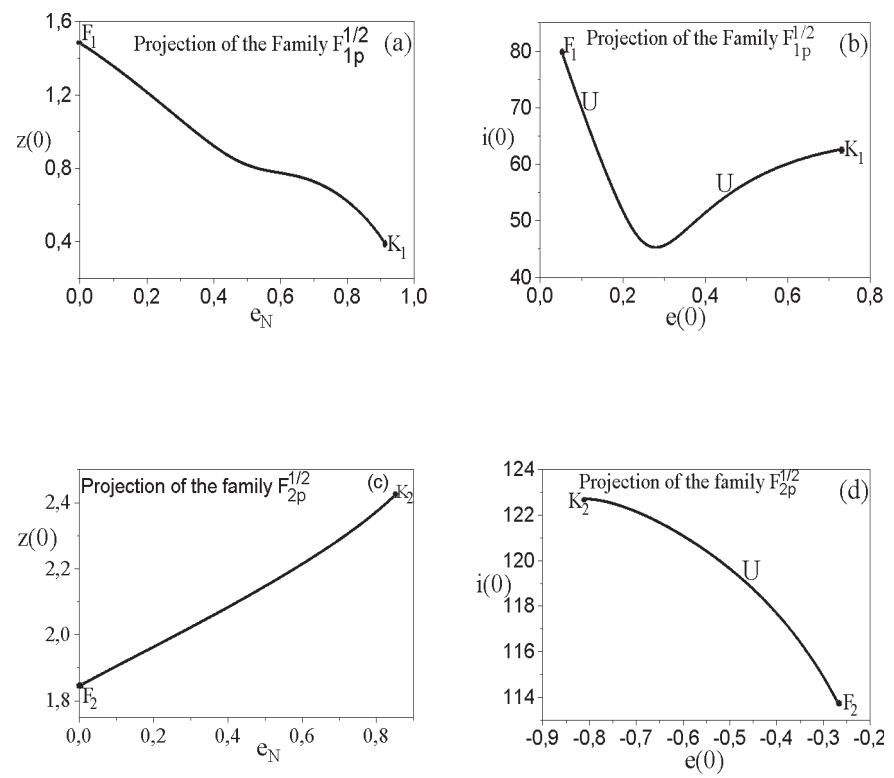

Fig. 5. a) Projection of the family $\mathrm{F}_{1 \mathrm{p}}^{1: 2}$ on the $z(0)-e_{\mathrm{N}}$ plane; and b) on the $e(0)-i(0)$ plane. c) Projection of the family $\mathrm{F}_{2 \mathrm{p}}^{1: 2}$ on the $z(0)-e_{\mathrm{N}}$ plane and $\left.\mathbf{b}\right)$ on the $e(0)-i(0)$ plane. The small body is at perihelion $(e(0)>0)$ at $t=0$ or at aphelion $(e(0)<0)$ at $t=0$. The starting points $\left(\mathrm{F}_{1}, \mathrm{~F}_{2}\right)$ and the terminating points $\left(\mathrm{K}_{1}, \mathrm{~K}_{2}\right)$ are also indicated.

The family $\mathrm{F}_{2 \mathrm{p}}^{1: 2}$ starts from the orbit $\mathrm{F}_{2}$ and terminates at the collision orbit $\mathrm{K}_{2}\left[e=0.81, i=122.71^{\circ}\right]$ with $e_{\mathrm{N}}=0.85$. Numerical difficulties appear for $e_{\mathrm{N}}>0.85$. In this family the small body is at aphelion at $t=0$ and the eccentricity increases continuously starting from $e=0.27$ and reaches the maximum value $e_{\max }=0.81$ at the collision orbit $\mathrm{K}_{2}$. The inclination starts from $i=113.80^{\circ}$ and reaches the maximum value $i_{\max }=122.71^{\circ}$ at the orbit $\mathrm{K}_{2}$. The stability of the family $\mathrm{F}_{2 \mathrm{p}}^{1: 2}$ is shown in Fig. 5d. The families $\mathrm{F}_{1 \mathrm{p}}^{1: 2}$ and $\mathrm{F}_{2 \mathrm{p}}^{1: 2}$ are both unstable. This means that we cannot have stable three dimensional motion of the small body in the 1:2 resonance for the 3D elliptic problem at high values of inclination.
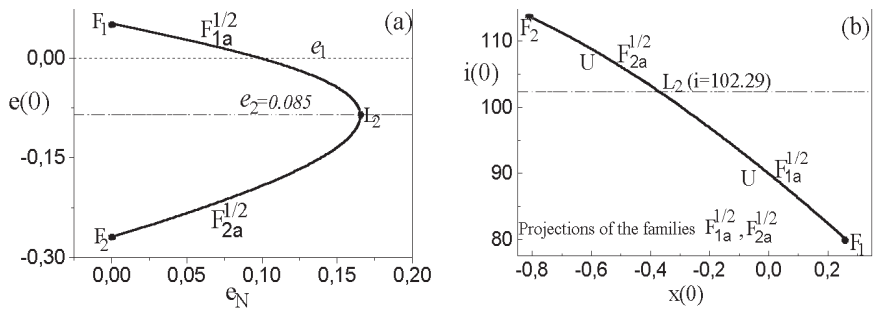

Fig. 6. Projections of the families $\mathrm{F}_{1 \mathrm{a}}^{1: 2}$ and $\mathrm{F}_{2 \mathrm{a}}^{1: 2}$ on the: a) $e(0)-$ $e_{\mathrm{N}}$ plane, where $e_{\mathrm{N}}$ is the eccentricity of Neptune and b) $x(0)-$ $i(0)$ plane. The starting and the terminating points of these families are also indicated. The negative eccentricity values denote only the initial position of the small body at aphelion.

\section{(ii) The families $F_{1 a}^{1: 2}, F_{2 a}^{1: 2}$}

In Figs. 6a-6b we present projections of the families $\mathrm{F}_{1 \mathrm{a}}^{1: 2}$, $\mathrm{F}_{2 \mathrm{a}}^{1: 2}$ on the $e(0)-e_{\mathrm{N}}$ and $x(0)-i(0)$ planes and their coincidence. The numerical computations showed that these families merge at a maximum eccentricity of Neptune $e_{\mathrm{N}}=0.165$. For the family $F_{1}^{1: 2}$ the small body is initially at perihelion while for the family $\mathrm{F}_{2 \mathrm{a}}^{1: 2}$ is initially at aphelion. In the family $\mathrm{F}_{1 \mathrm{a}}^{1: 2}$ the eccentricity of the small body decreases starting from $e_{0}=0.05$ until $e_{1}=0.0$ and then increases until it reaches the maximum value $e_{2}=0.085$ (Fig. 6a). In the family $\mathrm{F}_{2 \mathrm{a}}^{1: 2}$ the eccentricity decreases starting from $e_{0}=0.27$ until it reaches the minimum value $e_{2}=0.085$. In Fig. 6a the line $e_{1}=0.0\left(i=90^{\circ}\right)$ determines the point at which the small body passes from perihelion to aphelion in the family $\mathrm{F}_{1 \mathrm{a}}^{1: 2}$. The corresponding value of Neptune's eccentricity is: $e_{\mathrm{N}}=0.099$. This event causes a change in the initial value of $\omega$ and $\sigma$; it is $\omega=\pi / 2, \sigma=\pi$ now but the initial value for this family was $\omega=3 \pi / 2, \sigma=0$. Moreover, the line $e_{2}=0.085$ determines the point $\left(\mathrm{L}_{2}\right)$ where the merger of the two families mentioned above takes place. This corresponds to $e_{\mathrm{N}}=0.165$. In the family $\mathrm{F}_{1 \mathrm{a}}^{1: 2}$ the inclination increases starting from $i=80.02^{\circ}$ but in the family $\mathrm{F}_{2 \mathrm{a}}^{1: 2}$ the inclination decreases starting from $i=113.80^{\circ}$. The point at which these families meet is $i=102.29^{\circ}$ (Point: $\mathrm{L}_{2}$, Fig. 6b). The stability of these families is also shown in Fig. 6b. Both are unstable. So in this case there are no stable regions of motion in the phase space of the 3D elliptic problem.

There is one bifurcation point from the $3 \mathrm{D}$ circular family $\mathrm{A}_{2}$ to the $3 \mathrm{D}$ elliptic problem $(T=4 \pi)$. This is: $\mathrm{G}$ [ $e=0.037, i=101.66^{\circ}$ ]. So, one pair of families of periodic orbits of the $3 \mathrm{D}$ elliptic problem arises from this point. The pair is $G_{p}^{1: 2}, G_{a}^{1: 2}$. For the family $G_{p}^{1: 2}$ Neptune is at perihelion and for the family $\mathrm{G}_{\mathrm{a}}^{1: 2}$ it is at aphelion at $t=0$. These families of periodic orbits are of Type. For the family $\mathrm{G}_{\mathrm{p}}^{1: 2}$ we have $\sigma=\pi, \sigma_{z}=0, v=0$ and for the family $\mathrm{G}_{1 \mathrm{a}}^{1: 2}$ we have $\sigma=\pi$, $\sigma_{z}=0, v=\pi$. This is because the small body is at aphelion at $t=0$. For $\omega$ and $\Omega$ we have: $\omega=\pi$ and $\Omega=0$.

\section{(iii) The families $G_{p}^{1: 2}, G_{a}^{1: 2}$}

In Fig. 7 we present the families $\mathrm{G}_{\mathrm{p}}^{1: 2}$ and $\mathrm{G}_{\mathrm{a}}^{1: 2}$ in the space $\left(x_{0},(\mathrm{~d} y / \mathrm{d} t)_{0},(\mathrm{~d} z / \mathrm{d} t)_{0}\right)$. We remark here that these pictures are the projections of the families $\mathrm{G}_{\mathrm{p}}^{1: 2}$ and $\mathrm{G}_{\mathrm{a}}^{1: 2}$ on the $\left(x_{0}\right.$, $\left.(\mathrm{d} y / \mathrm{d} t)_{0},(\mathrm{~d} z / \mathrm{d} t)_{0}\right)$ space since a family of periodic orbits of 


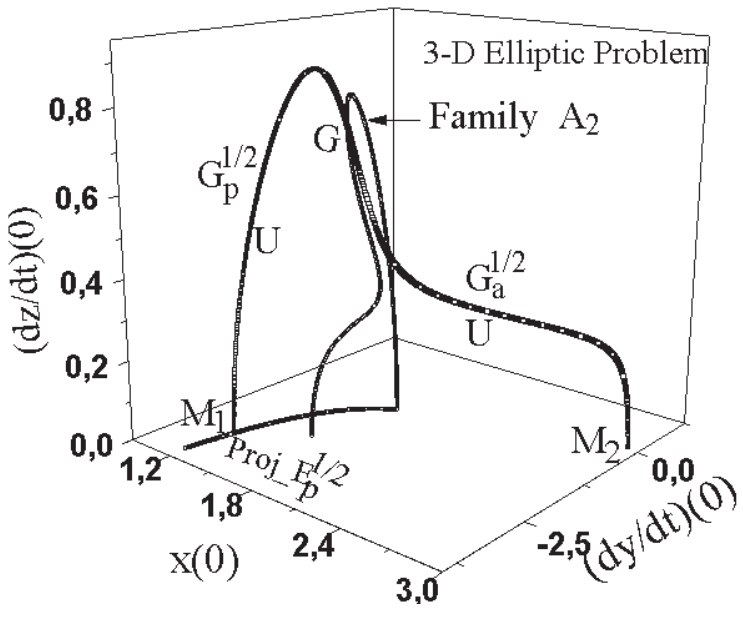

Fig. 7. Projections of the families $\mathrm{G}_{\mathrm{p}}^{1: 2}$ and $\mathrm{G}_{\mathrm{a}}^{1: 2}$ in the $x_{0}-\dot{y}_{0}-\dot{z}_{0}$ space, where $e_{\mathrm{N}}$ is the eccentricity of Neptune. The starting $(\mathrm{G})$ and the terminating points $\left(\mathrm{M}_{1}, \mathrm{M}_{2}\right)$ of these families are also indicated. The symbol "U" denotes instability of periodic orbits.

3D ER3BP is represented by a "characteristic curve" in the four-dimensional space mentioned above. The family $\mathrm{A}_{2}$ of the $3 \mathrm{D}$ circular restricted three-body problem is also presented. The point $G$ is the bifurcation point from the family $A_{2}$ to the 3D ER3BP. The curve in the $x_{0}-\dot{y}_{0}$ plane is the projection of the family $\mathrm{E}_{1 \mathrm{p}}^{1: 2}$ of the planar elliptic problem.

In Figs. $8 \mathrm{a}-\mathrm{b}$ we give the projections of the above families of the 3D ER3BP on the $x_{0}-e_{\mathrm{N}}$ and $e(0)-i(0)$ planes. The family $G_{\mathrm{p}}^{1: 2}$ starts from the point $\mathrm{G}$ and terminates at the point $\mathrm{M}_{1}\left[e=0.218, i=0^{\circ}\right]$ belonging to the family $\mathrm{E}_{\mathrm{p}}^{1: 2}$ of the planar periodic orbits. This point corresponds to $e_{\mathrm{N}}=$ 0.474 (Fig. 8a). In this family the eccentricity of the small body decreases, starting from $e=0.04$, until it reaches the minimum value $e=0.0$ (Point $\mathrm{N}_{1}$ ) and then increases continuously and reaches the maximum value $e_{\max }=0.218$ at the orbit $\mathrm{M}_{1}$ (Fig. 8b). On the other hand the inclination decreases continuously starting from $i=101.66^{\circ}$ and reaches the minimum value $i_{\min }=0^{\circ}$ at the orbit $\mathrm{M}_{1}$. Family $G_{p}^{1: 2}$ is unstable (Fig. 8b). In this family the small body is initially at aphelion $(e(0)<0)$. Note that at the point $\mathrm{N}_{1}\left[e=0.0, i=96.68^{\circ}\right]$ the small body passes from aphelion to perihelion. This causes a change in the initial value of $\omega$ and $\sigma$, viz. we now have $\omega=0, \sigma=0$ while the initial values for the family were $\omega=\pi, \sigma=\pi$. The values of $\sigma_{z}, v$ are then: $\sigma_{z}=0, v=0$.

We now come to the family $\mathrm{G}_{a}^{1: 2}$. In the same Figs. $8 \mathrm{a}-\mathrm{b}$ we give the projections of this family on the $x_{0}-e_{\mathrm{N}}$ and $e(0)-i(0)$ planes. In this family the small body is always at aphelion $(e(0)<0$, Fig. $8 \mathrm{~b})$ at $t=0$. This family starts from the point $\mathrm{G}$ and terminates at the point $\mathrm{M}_{2}\left[e=0.847, i=0.0^{\circ}\right]$ with $e_{\mathrm{N}}=0.976$ (Fig. 8a). This is a collision orbit of the planar elliptic problem. The eccentricity increases continuously in this family; it starts from low values $(e=0.04)$ and reaches the maximum value at the collision orbit $\mathrm{M}_{2}\left(e_{\max }=0.847\right)$. The inclination starts from $i=101.66^{\circ}$, reaches the maximum
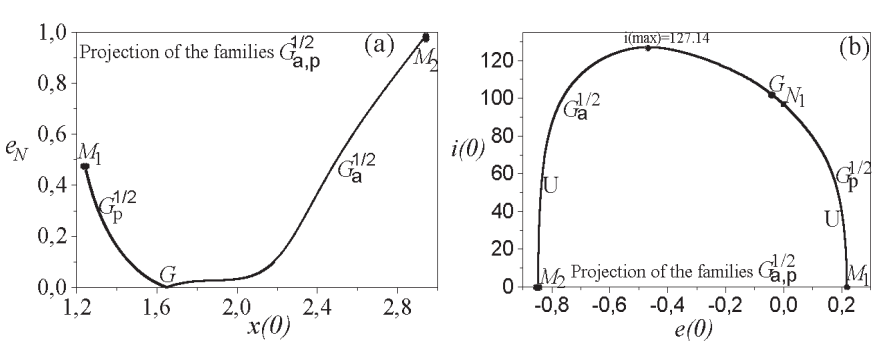

Fig. 8. a) Projections of the families $\mathrm{G}_{\mathrm{p}}^{1: 2}$ and $\mathrm{G}_{\mathrm{a}}^{1: 2}$ on the $x(0)-e_{\mathrm{N}}$ plane and $\mathbf{b}$ ) on the $e(0)-i(0)$ plane. The symbol " $\mathrm{U}$ " denotes instability. The negative values of the eccentricity denote only the initial position of the small body at aphelion. The starting point $(\mathrm{G})$ and the terminating points $\left(\mathrm{M}_{1}, \mathrm{M}_{2}\right)$ are also indicated.

value at $i=127.14^{\circ}$ and ends at zero (orbit $\mathrm{M}_{2}$ ). Family $\mathrm{G}_{\mathrm{a}}^{1: 2}$ is unstable (Fig. 8b).

\section{(iv) Comments}

In this section, we studied systematically the phase space structure of the 3D elliptic restricted three-body problem. In Sect. 3.2 it was found that in the 1:2 resonance the topology of the phase space in the elliptic planar model is separated by two pairs of families of unstable periodic orbits, one of them with very small eccentricities $(e=0.07)$ of the small body, which are close to each other. This means that there exist two unstable periodic orbits, close to each other, in the system Sun Neptune - small body $\left(e_{\mathrm{N}}=0.00912\right)$. Moreover, unstable motion appears in the 3D circular model for small values of the eccentricity. Exploring the phase space structure in the threedimensional elliptic problem, we found that the topology of the phase space is separated by three pairs of unstable periodic orbits; two of them start from very small eccentricities ( $e=0.04$ and $e=0.05$ ) and the third from a moderate eccentricity $(e=0.27)$. The inclination attains very high values in all cases $\left(i>80^{\circ}\right)$. It means that there exist six unstable periodic orbits, one close to one another, in the system Sun-Neptune-small body $\left(e_{\mathrm{N}}=0.00912\right)$. Consequently, large chaotic regions appear in the phase space for small and moderate values of the eccentricity when the eccentricity of Neptune is introduced in our model and the motion of the small body takes place in three-dimensional space.

Although the phase space of the 3D circular model was regular for $e>0.07$ and $i>0$, it becomes more complicated in the $3 \mathrm{D}$ elliptic case. Now it contains one more unstable fixed point (namely there is one more unstable periodic orbit with $e=0.27)$ and it is divided into two parts. The first part is $0.08<e<0.26$ and the second is $0.28<e<0.62$ (note the two bifurcation points at $e=0.07$ and $e=0.637$ from the planar circular to elliptic model - Fig. 1b). Also, the phase space is more difficult to explore when we take into consideration the bifurcation point $e=0.218\left(e_{\mathrm{N}}=0.474\right)$ from the $2 \mathrm{D}$ elliptic to the $3 \mathrm{D}$ elliptic problem and the chaotic regions are increased. Nesvorný \& Roig (2001) made a systematic study of the stability in the 1:2 resonant region. They found that the most stable region in the $1: 2$ resonance is at $e=0.3$; the chaotic structure of the resonant region at intermediate eccentricities $(0.1<e<0.4)$ is complex (see above p.113). Chaotic 
motion in the 1:2 resonance was also found by Gallardo \& Ferraz-Mello (1997). So, the discovery of new objects in the $1: 2$ resonant area is possible only in the above two areas because the phase space of the elliptic problem is dominated by regular orbits.

As far as the distribution of small bodies in the 1:2 resonant region is concerned, almost 18 objects are located near the N1:2 resonance ( $\pm 0.6 \mathrm{AU})$. One of them (1999 CL119) moves in nearly circular orbit and the rest of them have non-zero eccentricities. No objects with eccentricities $e \approx 0.07$ or $e \approx 0.60$ have yet been observed so perhaps they cannot stay at these regions. Our results are in good agreement with this observation. It is worth mentioning that there exist objects with $0.07<$ $e<0.1$ (e.g. 2001UP18 and 2001KA77). The majority of small bodies at the 1:2 resonant region has eccentricity values in the range $0.12<e<0.40$ and inclination $i<25^{\circ}$.

\section{Discussion}

In this paper we presented families of periodic orbits at the $1: 2$ resonance in the model of the $3 \mathrm{D}$ elliptic restricted threebody problem. Here we should state that only symmetric periodic orbits are considered in this paper. The main results are summarized below:

1) There exist two branches of elliptic orbits in the planar circular problem at the 1:2 resonance namely $\mathrm{I}_{1: 2}$ and $\mathrm{II}_{1: 2}$. The first one is unstable until $e=0.37$ where a perpendicular collision with Neptune occurs; then it becomes stable. The other one $\left(\mathrm{II}_{1: 2}\right)$ starts out stable; becomes unstable at $e=0.035$ and finally it again becomes stable at $e=0.96$. The critical points in which stability changes to instability and vice versa cause bifurcation in asymmetric periodic orbits (Voyatzis et al. 2004). The location of periodic orbits is important for obtaining the structure of the phase space.

2) Families of periodic orbits of the planar ER3BP bifurcate from the known families of the planar circular problem. All of them are unstable at the exterior resonance 1:2 with Neptune. In the case of the 1:2 resonance the majority of periodic orbits is unstable (Haghighipour et al. 2003).

3) There exist vertical critical orbits in the family $\mathrm{II}_{1: 2}$. Families of three-dimensional periodic orbits which bifurcate from vertical critical orbits of the planar problem are symmetric with respect to the $x z$-plane (type 1 ) or to the $x$-axis (type 2). Most of them are unstable.

4) Families of periodic orbits of the 3D ER3BP were computed. These families bifurcate from the known families of the $3 \mathrm{D}$ circular problem or from the vertical critical orbits of the planar elliptic problem. They are symmetric with respect to the $x z$-plane (type A) or to the $x$-axis (type B) and are generated by varying the eccentricity of Neptune $\left(e_{\mathrm{N}}\right)$. All of them are unstable. The instability of symmetric periodic orbits is related to chaotic motion which was also found by other authors, as we described in Sect. 1.

5) In the present study we dealt with realistic models with many degrees of freedom and computed families of symmetric periodic orbits in the spatial ER3BP for a specific value of mass and eccentricity of Neptune and found that instability is the dominant characteristic of these orbits at high-inclination values. But, as we saw in Sect. 4, there exist large regular regions in the phase space structure which can host small objects.

Acknowledgements. I am indebted to an anonymous referee for his useful remarks and suggestions which improved the paper. Many thanks to Prof. S. Ichtiaroglou for useful comments and discussions. My work was financially supported by the Hellenic Scholarship Foundation (I.K.Y.) and the scientific program "PYTHAGORAS", No. 21878, of the Greek Ministry of Education and EU.

\section{References}

Beaugé, C. 1994, Cel. Mech. \& Dyn. Astron., 60, 225

Beaugé, C., \& Ferraz-Mello, S. 1994, Icarus, 110, 239

Beaugé, C., Ferraz-Mello, S., \& Michtchenko, T. A. 2003, ApJ, 593, 1124

Broucke, R. A. 1968, Periodic Orbits in the Elliptic Restricted Three Body problem, JPL Technical Report, 32

Broucke, R. A. 1969, AIAA, 7, 1003

Broucke, R. A. 2001, Celest. Mech. and Dyn. Astron., 81, 321

Celletti, A., Chessa, A., Hadjidemetriou, J. D., \& Valsecchi, G. B. 2002, Celest. Mech. \& Dyn. Astron., 83, 239

Chiang, E. I., \& Jordan, A. B. 2002, AJ, 124, 3430

Duncan, M. J., Levison, H. F., \& Budd, S. M. 1995, AJ, 110 (6), 1995, 3073

Ferraz-Mello., S., Beaugé, C., \& Michtchenko, T. A. 2003, Cel. Mech. \& Dyn. Astron., 87, 99

Gallardo, T., \& Ferraz-Mello, S. 1997, AJ, 113, 863-875

Hadjidemetriou, J. D. 1975, Cel. Mech., 12, 255

Hadjidemetriou, J. D. 1988, Cel. Mech., 43, 371

Hadjidemetriou, J. D. 1992, Cel. Mech., 53, 151

Hadjidemetriou, J. D. 1993, Cel. Mech \& Dyn. Astr., 56, 201

Hadjidemetriou, J. D., \& Ichtiaroglou, S. 1984, A\&A, 131, 20

Haghighipour, N., Couetdic, J., Varadi, F., \& Moore, W. B. 2003, ApJ, 596,1332

Hénon, M., II 1965, Ann. Astrophys., 28, 992

Hénon, M. 1973, A\&A, 28, 415

Hénon, M. 1997, Generating Families in the Restricted Three-Body Problem (New York: Springer)

Holman, M. J., \& Wisdom, J. 1993, AJ, 105, 1987

Ichtiaroglou, S., \& Michalodimitrakis, M. 1980, A\&A, 81, 30

Ichtiaroglou, S., katopodis, K., \& Michalodimitrakis, M. 1978, A\&A, 70,531

Jewitt, D. 1999, Annu. Rev. Planet. Sci., 27, 287-312

Jewitt, D., \& Luu, J. X. 2000, Physical Nature of the Kuiper Belt, in Protostars and Planets IV, ed. V. Mannings, A. P. Boss, \& S. S. Russell (Univ. Az. press), 1201

Knežević, Z., Milani, A., Farinella, P., Froeschlé, Ch., \& Froeschlé, C. 1991, Icarus, 93, 316

Kotoulas, Th. A., \& Hadjidemetriou, J. D. 2002, Earth, Moon \& Planets, 91, 63 (KH2002)

Kribbel, J., \& Dvorak, R. 1988, Cel. Mech., 43, 391

Lee, M. H., \& Peale, S. J. 2003, Extrasolar Planets and Mean-Motion Resonances, in Scientific Frontiers in Research on Extrasolar Planets, ed. D. Deming, \& S. Seager (San Francisco: ASP), ASP Conf. Ser., 294, 197

Lee, M. H. 2004, ApJ, accepted for publication [arXiv: astro-ph/0401410]

Levison, H. F., \& Duncan, M. J. 1993, ApJ, 406, L35

Malhotra, R. 1996, AJ, 111 (1), 1996, 504-516 
Markellos, V. V., Goudas, C. L., \& Katsiaris, G. A. 1981, Bifurcations of planar to three-dimensional periodic orbits in the restricted three-body problem, in Investigating the Universe, ed. F. D. Kahn (D. Reidel Publishing Company), 321

Melita, M. D., \& Brunini, A. 2000, Icarus, 147, 205

Message, P. J. 1958, AJ, 63, 443

Michalodimitrakis, M. 1979, A\&A, 76, 6

Morbidelli, A. 1999, Cel. Mech. \& Dyn.Astr., 72, 129

Morbidelli, A., Thomas, F., \& Moons, M. 1995, Icarus, 118, 322

Nesvorný, D., \& Roig, F., II 2001, Icarus, 150, 104

Roy, A. E. 1982, Orbital Motion (Adam Hilger, $2^{\text {nd }}$ ed.)

Schubart, J. 1964, Long-period effects in nearly commensurable cases of the restricted three-body problem, Smithson. Astrophys. Obs. Spec. Rep., 149

Simó, Carles. 1995, Hamiltonian Systems with Three or More Degrees of Freedom (Kluwer Academic Publishers)

Skokos, Ch. 2001, Physica D, 159, 155
Szebehely, V. 1967, Theory of Orbits (Academic Press)

Tsiganis, K., Varvoglis, H., \& Hadjidemetriou, J. D. 2002a, Icarus, 155,454

Tsiganis, K., Varvoglis, H., \& Hadjidemetriou, J. D. 2002b, Icarus, 159,284

Varadi, F. 1999, AJ, 118, 2526

Voyatzis, G., Kotoulas, T., \& Hadjidemetriou, J. D. 2004, Cel. Mech. \& Dyn. Astron., in press

Winter, O. C., \& Murray, C. D. 1994a, Atlas of the Planar, Circular, Restricted Three-Body Problem I. Internal Orbits, QMW Maths Notes, London

Winter, O. C., \& Murray, C. D. 1994b, Atlas of the Planar, Circular, Restricted Three-Body Problem II. External Orbits, QMW Maths Notes, London

Winter, O. C., \& Murray, C. D., II 1997, A\&A, 328, 399

Zagouras, C., \& Markellos, V. V. 1977, A\&A, 59, 79 